von Diagnosen und Behandlungssituationen grundsätzlich regeln, in einem speziellen Teil werden die für die Codierung einzelner Krankheiten, Krankheitsgruppen und Behandlungssituationen notwendigen Vorgaben gemacht.

\section{MMW Kommentar}

Die Einführung der AKR erfolgt zum 1. Juli 2010 und dann zunächst nur in Testregionen. Die aktuelle Version enthält elf allgemeine und 59 spezielle Codierrichtlinien. Die allgemeinen Codierrichtlinien sind dreistellig und beginnen immer mit dem Großbuchstaben „A“. Danach folgt eine fortlaufende Nummerierung von o1 bis 11. Der Teil B mit den speziellen Codierrichtlinien ist in 18 Kapitel untergliedert, die zur leichteren Orientierung in Nummerierung und Text mit den Kapiteln der ICD-10-GM identisch sind. Die speziellen Codierrichtlinien sind fünfstellig. Sie beginnen immer mit dem Großbuchstaben „B“. Danach folgt an zweiter und dritter Stelle die Kapitelnummer von 01 bis 21. Die vierte und fünfte Stelle ist eine, in jedem Kapitel neu mit oo (Doppel-Null) beginnende, fortlaufende Nummerierung. Zwischen der jeweiligen Kapitelnummer und der fortlaufenden Nummerierung wurde auf ein Trennzeichen verzichtet, um Verwechslungen mit den ICD-Codes zu vermeiden. Neu sind u.a. Plausibilitätskriterien zur Validierung der Diagnosestellung. Sie beziehen sich ausschließlich auf gesicherte Diagnosen mit dem Zusatzkennzeichen "G" und benennen bestimmte Mindestanforderungen, die zur Codierung einer Diagnose vorliegen müssen.

Dies können sowohl bestimmte diagnostische Verfahren als auch die (Mit-)Behandlung durch einen speziellen Facharzt sein. So kann bei der Codierung einer zerebral transitorisch ischämischen Attacke (TIA) ein MRT oder ein CT gefordert sein. Die AKR werden regelmäßig überarbeitet, wobei nicht nur die Änderungen der ICD-10-GM und des EBM berücksichtigt werden, sondern auch die Veränderungen der Medizin sowie die Weiterentwicklung des Risikostrukturausgleiches und des Klassifikationssystems nach \$87a SGB V.

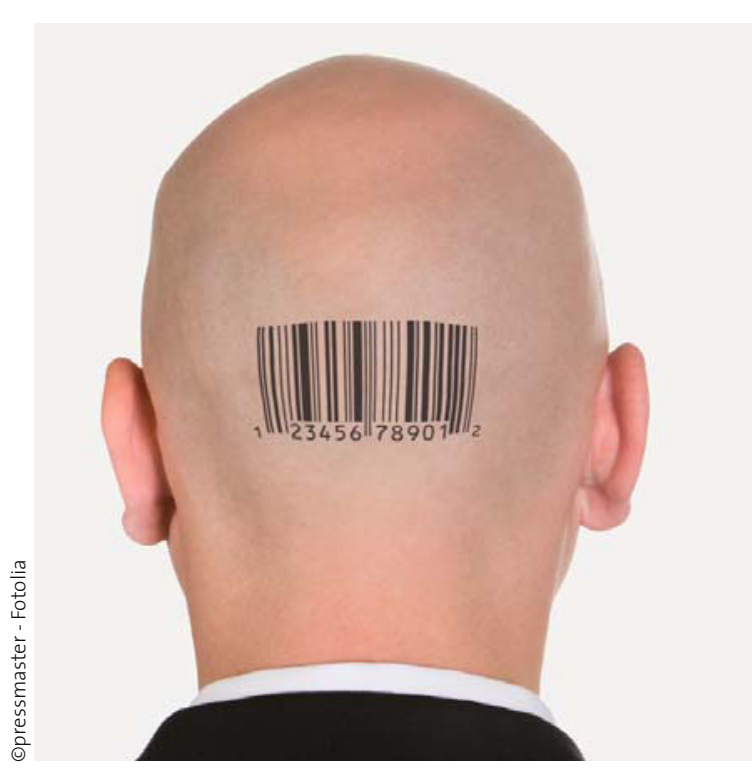

AKR sollen bald die Codierung der Diagnosen zur Abrechnung regulieren.

Ob die daraus ggf. resultierenden Kostensteigerungen über eine Anpassung der morbiditätsbedingten Gesamtvergütung abgedeckt werden, ist bisher allerdings noch nicht geregelt.

\title{
Bundessozialgericht: Honorarverteilung war rechtswidrig
}

— Nach dem Sozialgericht Stuttgart (SG) und dem Landessozialgericht (LSG) Baden-Württemberg hat nun auch das Bundessozialgericht (BSG) den Honorarverteilungsvertrag (HVV) der ehemaligen KV Nordwürttemberg seit dem 1.4.2005 für rechtswidrig erklärt.

Die KV Baden-Württemberg muss nunmehr die noch offenen Honorarbescheide unter Beachtung der Rechtsauffassung des BSG neu bescheiden.

Die Entscheidung des BSG basiert auf der Feststellung, dass ab diesem Zeitpunkt Regelleistungsvolumina (RLV) mit festen
Punktwerten vorgeschrieben waren und deshalb diejenigen Ärzte, die durch Regelungen mit floatenden Punktwerten negativ betroffen waren, eine Nachvergütung erhalten müssen. Eine Verböserung ist gleichzeitig ausgeschlossen, d.h. niemand muss zurückzahlen, wenn eine Neuberechnung ein schlechteres finanzielles Ergebnis nach sich zieht.

\section{MMW Kommentar}

Offen lässt das Urteil allerdings, ob es auch für andere KV-Regionen Gültigkeit hat. Floatende Punktwerte gab es nämlich zum 1.4.2005 nicht nur in ganz BadenWürttemberg, sondern auch in nahezu allen anderen Kassenärztlichen Vereinigungen. Lediglich die KV Hessen hatte sich seinerzeit exakt an die gesetzlichen Vorgaben des $\$ 85$ Abs. 4 SGB V gehalten, der eindeutig vorschreibt, dass $a b 2005$ ein Anspruch auffeste Punktwerte im RLV bestand. 